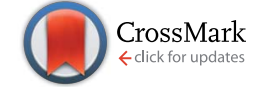

Cite this: RSC Adv., 2017, 7, 224

Received 11th October 2016 Accepted 5th November 2016

DOI: 10.1039/c6ra24927b

www.rsc.org/advances

\section{Porous hollow carbon nanofibers derived from multi-walled carbon nanotubes and sucrose as anode materials for lithium-ion batteries $\dagger$}

\author{
Wanwan Lei, ${ }^{\text {abc }}$ Sheng Liu*ab and Wen-Hua Zhang ${ }^{\text {ab }}$ \\ Porous hollow carbon nanofibers with tunable shell thicknesses from 2.5 to $13.5 \mathrm{~nm}$ have been synthesized \\ via a hydrothermal approach by using multi-walled carbon nanotubes as the scaffolds and sucrose as \\ carbon source. The thin, porous and non-graphitic carbon shells are revealed by X-ray diffraction, \\ Raman spectra, high-resolution transmission electron microscopy, and nitrogen adsorption isotherms. In \\ comparison with the pristine multi-walled carbon nanotubes, the as-prepared porous hollow carbon \\ nanofibers exhibit the enhanced reversible capacities, cycling stabilities and rater performances when \\ evaluated as anode materials for lithium-ion batteries.
}

\section{Introduction}

One-dimensional carbon nanomaterials including carbon nanotubes (CNTs) and carbon nanofibers (CNFs) have been widely investigated as electrode materials for lithium-ion batteries (LIBs). ${ }^{1-8}$ Compared to the traditional carbon electrode materials, e.g., graphite or active carbon, one-dimensional carbon nanomaterials exhibit differences not only in dimensionality and morphology but also in the distribution of saturated bonds on the surface. The unique structural feature produces huge superiority in forming conductive networks at a rather low loading due to the large aspect ratio and high electrical conductivity. ${ }^{9}$ The excellent mechanical property raises vast potential to buffer the volumetric change in the electrode during charging/discharging process. ${ }^{10}$ The onedimensional carbon nanomaterials also held promises to scaffold a wide variety of nanoscale electrode materials such as elements and metal oxides, as well as work as lightweight and flexible current collectors. ${ }^{11,12}$

Although the multi-walled carbon nanotubes (MWCNTs) exhibit a superior capacity to graphite, tremendous attempts to tailor the pristine MWCNTs for further improving their electrochemical properties are still under way, e.g., acid oxidation, ${ }^{13-15}$ ball milling, ${ }^{\text {16-18 }}$ cutting, ${ }^{19,20}$ drilling holes, ${ }^{21}$ opening cap, ${ }^{22}$ etc. Recently, non-graphitic carbon nanotubes, in

${ }^{a}$ Institute of Chemical Materials, China Academy of Engineering Physics, P. O. Box 919-301, Mianyang 621900, P. R. China. E-mail: sliu@caep.cn; Tel: +86-28-65726193 ${ }^{b}$ Sichuan Research Center of New Materials, 596 Yinhe Road, Shuangliu District, Chengdu 610200, China

${ }^{c}$ School of Chemistry and Chemical Engineering, Southwest University, 2 Tiansheng Road, Bebei District, Chongqing 400715, China

$\dagger$ Electronic supplementary information (ESI) available. See DOI: $10.1039 / \mathrm{c} 6 \mathrm{ra} 24927 \mathrm{~b}$ comparison with the traditional graphitic carbon nanotubes, demonstrate enhanced lithium storage capability due to their distinct tube wall structure which is in abundance with small stacked graphite sheets and in favor of providing much more active sites. ${ }^{23,24}$ Meanwhile, fabrication of thin hollow CNFs is still rare, ${ }^{25}$ since most of the reported hollow CNFs have a relatively large particle size of over $100 \mathrm{~nm} \cdot{ }^{\mathbf{4 , 2 6 - 2 8}}$ Therefore, coating a non-graphitic carbon layer on MWCNTs to construct thin hollow CNFs with diameters less than $100 \mathrm{~nm}$ is highly expected to enhance the electrochemical properties.

Herein, thin porous hollow carbon nanofibers (PHCNFs) with diameters less than $100 \mathrm{~nm}$ have been prepared via a hydrothermal approach using MWCNTs as the scaffolds. Derived from sucrose, the porous non-graphitic carbon shells with tunable thicknesses are homogenously coated on MWCNTs. Microstructures of PHCNFs are investigated in detail by X-ray diffraction, Raman spectra, high-resolution transmission electron microscopy, and nitrogen adsorption isotherms, etc. When evaluated as the anode in coin-type LIBs, the variation of shell microstructures such as the thickness and the pore structure significantly impacts on the electrochemical properties.

\section{Experimental}

\subsection{Preparation of PHCNFs}

All the commercial chemicals were of analytical grade. Firstly, a certain amount of sucrose was dissolved in $88 \mathrm{ml}$ of deionized water. Secondly, $10 \mathrm{mg}$ of carboxyl functionalized MWCNTs (XFM27, purity > 95\%, Nanjing XFNANO Materials Tech Co., Ltd, China) were dispersed in the above solution by ultrasonic treatment for $30 \mathrm{~min}$. Thirdly, the resultant dispersion was transferred into a $100 \mathrm{ml}$ Teflon-lined stainless steel autoclave and kept at $190{ }^{\circ} \mathrm{C}$ for $5 \mathrm{~h}$. After naturally cooled to room 
temperature, the black deposit was collected by centrifugation and washing by deionized water for three times. Lastly, the raw products were dried under vacuum at $60{ }^{\circ} \mathrm{C}$ overnight. The products, derived from $0.10,0.25$ and $1.00 \mathrm{~g}$ of sucrose, were denoted as PHCNFs-1, PHCNFs-2 and PHCNFs-3, respectively.

\subsection{Characterizations}

Powder XRD patterns were collected using a Maxima 7000 diffractometer with graphite-monochromatized $\mathrm{Cu} \mathrm{K} \alpha$ radiation $(\lambda=1.5418 \AA)$. Raman spectra were recorded on a Renishaw Invia Raman spectrometer with an excitation laser wavelength of $532 \mathrm{~nm}$. High-resolution transmission electron microscope (HRTEM) images were analyzed using a JEM-2100 (JEOL, Japan). The Brunauer-Emmett-Teller (BET) surface area and pore structures were tested by nitrogen adsorption-desorption analysis using a ASAP-2020 apparatus (Micromeritics, USA).

\subsection{Electrochemical measurements}

Work electrodes were fabricated by coating a slurry mixture of MWCNTs or PHCNFs (active material), carbon black (Super-P) and sodium carboxyl methyl cellulose (CMC) (with a mass ratio of $8: 1: 1$ ) onto a $\mathrm{Cu}$ foil. A lithium foil was used as the counter electrode. The value of mass loaded on the area was about $0.80 \mathrm{mg} \mathrm{cm}^{-2}$. The electrolyte was a solution of $1 \mathrm{M} \mathrm{LiPF}_{6}$ in mixed solvents of ethylene carbonate (EC) and diethyl carbonate (DEC) with a volume ratio of $1: 1$. Electrochemical measurements were conducted by coin-type cells (CR2032) which were assembled in an argon filled glove box (Dellix, China). Galvanostatic charge-discharge tests were carried out on a battery tester (Land CT2001A, China) in a voltage of 0.01$3 \mathrm{~V}$ at various current densities. Electrochemical impedance spectroscopy (EIS) was performed on an electrochemical workstation CHI 660D (Shanghai, China) in a frequency range from $0.1 \mathrm{~Hz}$ to $1.0 \times 10^{5} \mathrm{~Hz}$.

\section{Results and discussion}

Sucrose is thought to be one of the best carbon sources for uniformly coating undoped carbon layers on the other nanomaterials such as $\mathrm{LiFePO}_{4},{ }^{29}$ graphite microfiber, ${ }^{30} \mathrm{Fe}_{3} \mathrm{O}_{4},{ }^{31}$ etc. Furthermore, the molecular formula of sucrose is $\mathrm{C}_{12} \mathrm{H}_{22} \mathrm{O}_{11}$. It doesn't contain any nitrogen, sulfur, phosphorus, or other doping elements. Therefore, sucrose is selected as pure carbon source for coating MWCNTs in this work.

Wide-angle XRD patterns of MWCNTs and PHCNFs are displayed in Fig. 1a. The prominent diffraction peaks centered at $26^{\circ}$ are ascribed to (002) planes of graphitic carbon. Apparently, intensities of the (002) peaks become weaker with increasing thicknesses of carbon shells from the pristine MWCNTs to PHCNF-3, indicative of the low crystallinity and amorphous structure of the overcoated carbon shells. ${ }^{23}$ The values of $d_{002}$ are summarized in Table 1. PHCNF-3 has a smallest $d_{002}$ value in all the samples, hinting a largest interlayer distance.

To further reveal the microstructure and crystalline ordering, Raman spectra of MWCNTs and PHCNFs are presented in Fig. 1b. Two strong peaks at $\sim 1341$ and $\sim 1573 \mathrm{~cm}^{-1}$ are clearly viewed, which correspond to the disorder carbon "D-band" and the graphite "G-band", respectively. ${ }^{12}$ The relative intensity ratio of D-band to G-band $\left(I_{\mathrm{D}} / I_{\mathrm{G}}\right)$ is proportional to disorder carbonaceous materials and grows in value with increasing defect sites. As shown in Table $1, I_{\mathrm{D}} / I_{\mathrm{G}}$ value of the pristine MWCNTs is smallest (0.74), which confirms a most ordered graphitic structure. PHCNF-1 exhibits a largest $I_{\mathrm{D}} / I_{\mathrm{G}}$ value $(0.86)$, resulting from the most disordered stacking of ultrathin carbon layers. With the thickness increase of non-graphitic carbon shells, the disorder degree of carbon shells obviously decreases, which can be ascribed to the release of micro-strains between nongraphitic carbon shells and the pristine MWCNTs. PHCNF-2 and PHCNF-3 deliver the similar $I_{\mathrm{D}} / I_{\mathrm{G}}$ values $(0.76$ and 0.78 , respectively) to that of MWCNTs, demonstrating a favorable crystalline ordering.

The thicknesses of non-graphitic carbon shells are determined by sucrose concentrations in the hydrothermal reactions. As shown in Fig. 2, HRTEM are utilized to examine the

Table 1 Characterization parameters of MWCNTs and PHCNFs

\begin{tabular}{llll}
\hline Sample & $d_{002}(\mathrm{~nm})$ & $I_{\mathrm{D}} / I_{\mathrm{G}}$ & Shell thickness $(\mathrm{nm})$ \\
\hline MWCNTs & 0.345 & 0.74 & 0 \\
PHCNF-1 & 0.344 & 0.89 & $\sim 2.5$ \\
PHCNF-2 & 0.344 & 0.76 & $\sim 5.0$ \\
PHCNF-3 & 0.341 & 0.78 & $\sim 13.5$
\end{tabular}
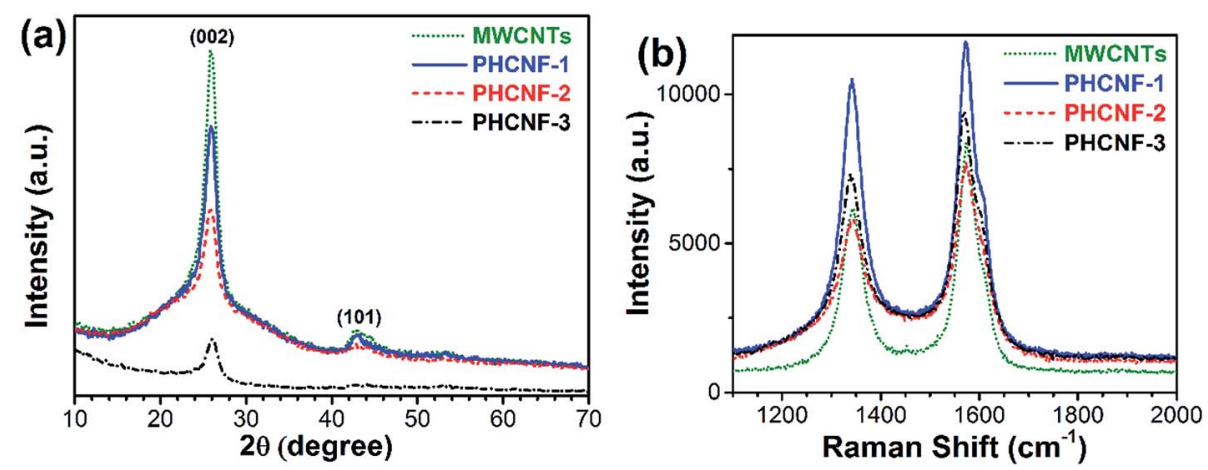

Fig. 1 (a) XRD patterns and (b) Raman spectra of MWCNTs, PHCNF-1, PHCNF-2, and PHCNF-3. 

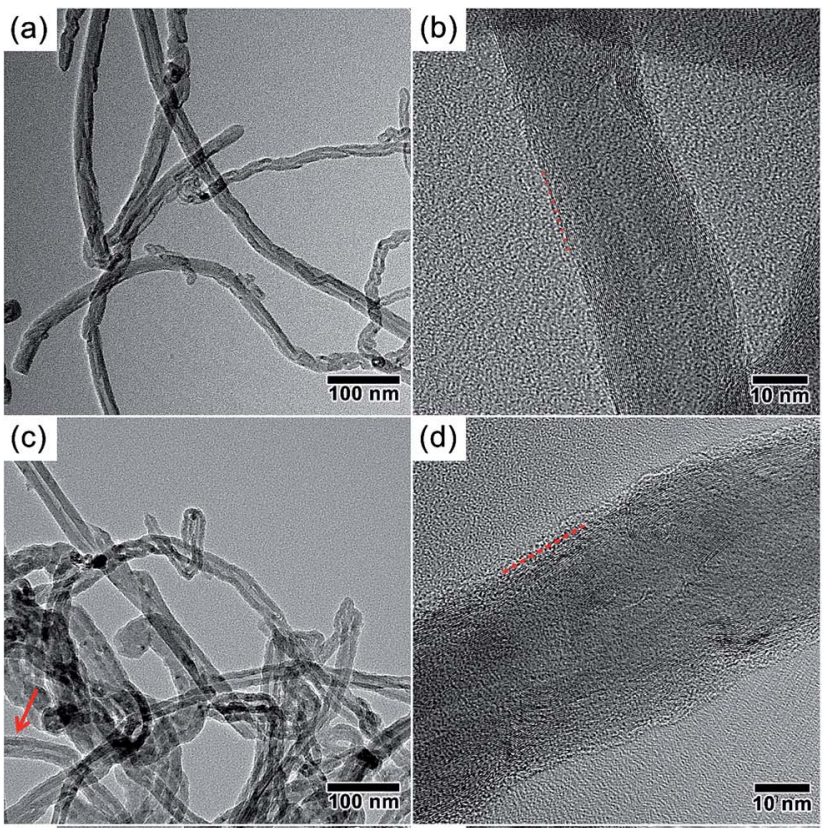

(d)
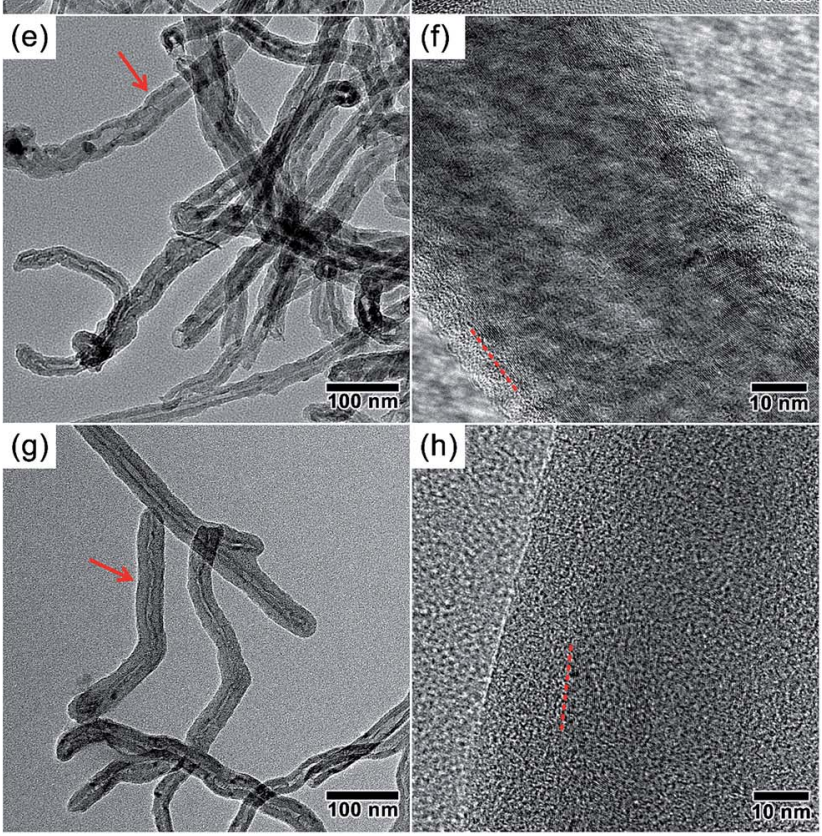

Fig. 2 TEM and HRTEM images of ( $a$ and $b$ ) the pristine MWCNTs, (c and d) PHCNF-1, (e and f) PHCNF-2, and ( $g$ and h) PHCNF-3. The arrows indicate the non-graphitic carbon shells, and the dashed lines indicate the interfaces between the porous shells and MWCNTs.

microstructures and surface changes of all the samples. The pristine MWCNTs (outer diameter: $\sim 25 \mathrm{~nm}$, inner diameter: $\sim 5$ $\mathrm{nm}$ ) show the graphitic multi-walled structures and clean surfaces (Fig. 2a and b). Once hydrothermally treated in sucrose solutions with a variety of concentrations, the clearly visible non-graphitic carbon layers with various thicknesses are homogeneously formed on the surfaces of MWCNTs. With an increase of sucrose concentrations, the shell thickness of PHCNF-1, PHCNF-2, and PHCNF-3 reaches to $\sim 2.5, \sim 5.0$, and $\sim 13.5 \mathrm{~nm}$, respectively (Table 1). Different from the rough surfaces of PHCNF-1, PHCNF-3 displays an apparently smoother surface, which suggests a more ordered stacking of carbon lattices. The analyses of HRTEM validate the results of XRD and Raman.

The surface area and pore structure of carbon nanomaterials play a vital role in their lithium storage properties. To analyze these characteristics, nitrogen adsorption-desorption isotherms and pore size distributions are recorded in Fig. 3. As summarized in Table 2, the BET specific surface areas of PHCNF-1 and PHCNF-2 dramatically rise to 225.5 and $285.8 \mathrm{~m}^{2} \mathrm{~g}^{-1}$, with respective to $118.4 \mathrm{~m}^{2} \mathrm{~g}^{-1}$ for the pristine MWCNTs. But the $t$-plot micropore areas only slightly increase from 22.3 to 28.0 and $38.5 \mathrm{~m}^{2} \mathrm{~g}^{-1}$. In combination with the data of $t$-plot micropore volumes and BJH mesopore volumes, the increased BET specific surface areas of PHCNF-1 and PHCNF-2 is mainly derived from the mesopore structures of the non-graphitic carbon shells. According to the same analytic method, PHCNF-3 is revealed to possess an absolutely different pore structure, which contains moderate mesopores but abundant micropores. According to the calculation of $D_{\text {Average }}=4 V_{\text {Total }} / S_{\text {BET }}$ using the cylinder model, the average pore diameter $D_{\text {Average }}$ of MWCNTs is estimated to be $7.66 \mathrm{~nm}$ (Table 2), which is corresponding to the inner wall diameter of MWCNTs. With thickness increase of non-graphitic carbon shells, the average pore diameters $D_{\text {Average }}$ of PHCNFs gradually decrease from 6.05 to $2.87 \mathrm{~nm}$, which is in good agreement with the previous analyses of specific surface areas and pore volumes. In brief, the porous and non-graphitic carbon overlayers ensure better intercalation and diffusion of lithium ions into hollow carbon nanofibers.

Fig. 4a displays 1st, 2nd, 5th, and 50th discharge/charge voltage profiles of PHCNF-2 electrode at a current density of $0.05 \mathrm{~A} \mathrm{~g}^{-1}$. All the curves are featureless, and no evident voltage plateau is seen, which is a representative characteristics of hard carbon materials. ${ }^{32-34}$ The PHCNF-2 electrode delivers an initial specific discharge and charge capacity of 456.2 and $398.0 \mathrm{~mA} \mathrm{~h}$ $\mathrm{g}^{-1}$, suggesting an initial coulombic efficiency of $87 \%$. The relatively low coulombic efficiency in the first cycle could result from the irreversible formation of solid electrolyte interface (SEI) layers on the PHCNF-2 anode due to the electrolyte decomposition. After cycling, the specific discharge/charge capacities decay gradually. After 50 cycles, the discharge and charge capacities fade to 297.3 and $293.7 \mathrm{~mA} \mathrm{~h} \mathrm{~g}^{-1}$, and the coulombic efficiency returns to nearly $99 \%$. The discharge/ charge voltage profiles of PHCNF-2 electrode at various rate are presented in Fig. $4 \mathrm{~b}$. As the rate increases, the reversible capacities of the PHCNF-2 electrode dramatically fade from 0.05 to $1 \mathrm{~A} \mathrm{~g}^{-1}$, and then moderately decay from 1 to $5 \mathrm{~A} \mathrm{~g}^{-1}$.

The cycling stabilities of MWCNTs and PHCNFs are displayed in Fig. 4c. In the first 4 cycles, the specific discharge capacities of MWCNTs, PHCNF-2 and PHCNF-3 reduce rapidly, but PHCNF-1 make a smaller variation. After 50 cycles, MWCNTs, PHCNF-1 and PHCNF-3 only deliver the discharge capacities of $216.3,269.7$, and $215.8 \mathrm{~mA} \mathrm{~h} \mathrm{~g}^{-1}$, respectively. As a whole, PHCNF-2 shows a most superior cyclability in all the samples at a low current density of $0.05 \mathrm{~A} \mathrm{~g}^{-1}$.

To evaluate the rate capability, all the electrodes of MWCNTs and PHCNFs are discharged and charged stepwise at a current 

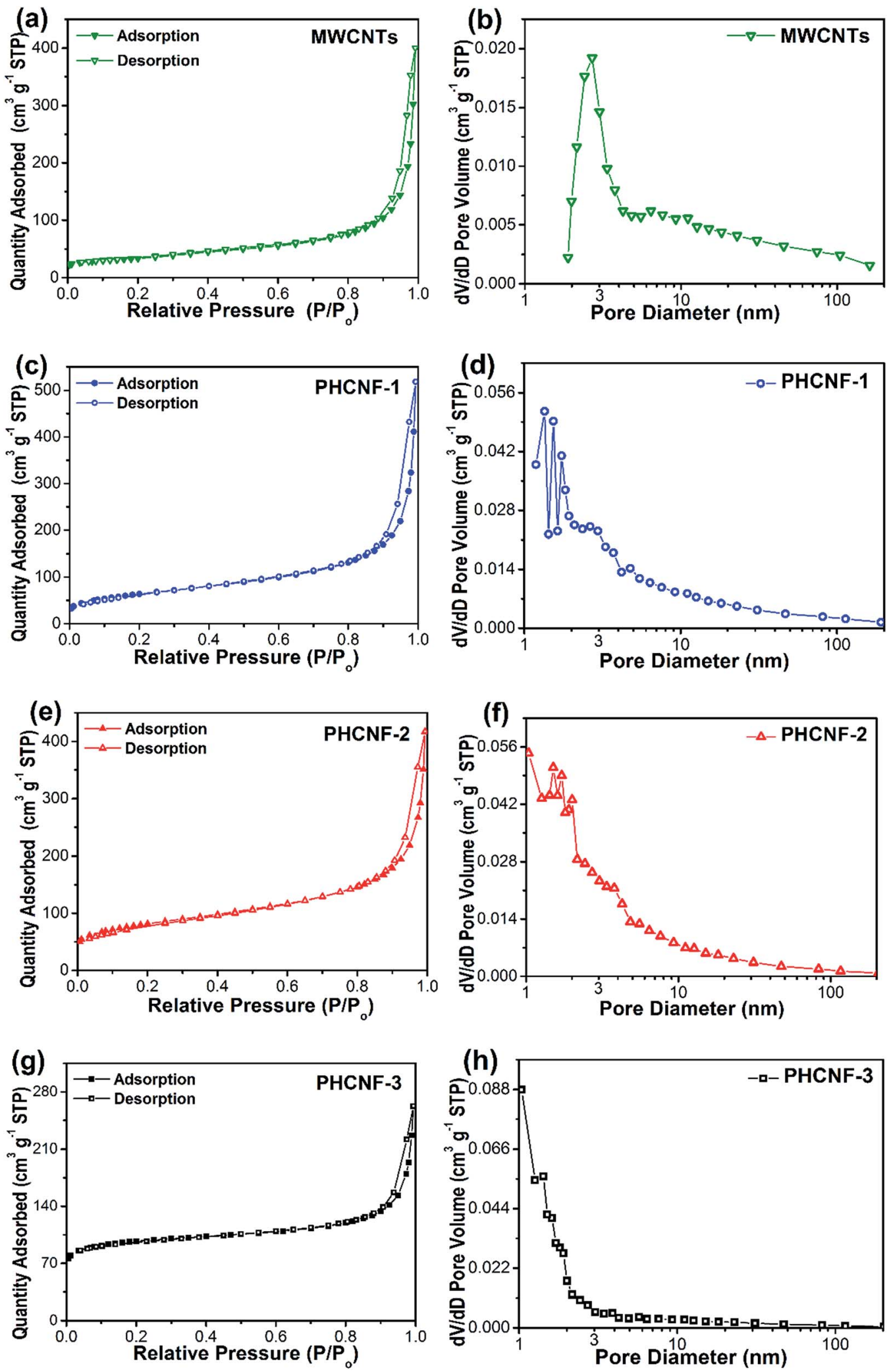

Fig. 3 Nitrogen adsorption-desorption isotherms and pore size distributions of (a and b) the pristine MWCNTs, (c and d) PHCNF-1, (e and f) PHCNF-2, and ( $g$ and h) PHCNF-3.

density from 0.05 to $5 \mathrm{~A} \mathrm{~g}^{-1}$, and then the rate returns to $0.05 \mathrm{~A}$ $\mathrm{g}^{-1}$ (Fig. 4d). The final reversible capacities of the PHCNF-2 electrode reach to $387.4,269.1,214.3,168.5,160.3$, and 146.7
$\mathrm{mA} \mathrm{h} \mathrm{g}{ }^{-1}$ at $0.05,0.1,0.2,0.5,1$, and $2 \mathrm{~A} \mathrm{~g}^{-1}$, respectively. In contrast, the MWCNT electrode only delivers the final reversible capacities of 266.5, 213.6, 170.4, 130.9, 105.4, and $77.8 \mathrm{~mA} \mathrm{~h} \mathrm{~g}^{-1}$ 
Table 2 Nitrogen adsorption analyses of MWCNTs and PHCNFs

\begin{tabular}{|c|c|c|c|c|c|c|}
\hline Sample & $S_{\text {BET }}\left(\mathrm{m}^{2} \mathrm{~g}^{-1}\right)$ & $S_{\text {Micro }}\left(\mathrm{m}^{2} \mathrm{~g}^{-1}\right)$ & $V_{\text {Total }}\left(\mathrm{cm}^{3} \mathrm{~g}^{-1}\right)$ & $V_{\text {Micro }}\left(\mathrm{cm}^{3} \mathrm{~g}^{-1}\right)$ & $V_{\text {Meso }}\left(\mathrm{cm}^{3} \mathrm{~g}^{-1}\right)$ & $D_{\text {Average }}(\mathrm{nm})$ \\
\hline MWCNTs & 118.4 & 22.3 & 0.61 & 0.01 & 0.23 & 7.66 \\
\hline PHCNF-1 & 225.5 & 28.0 & 0.79 & 0.01 & 0.34 & 6.05 \\
\hline PHCNF-3 & 331.7 & 260.0 & 0.33 & 0.12 & 0.24 & 2.87 \\
\hline
\end{tabular}
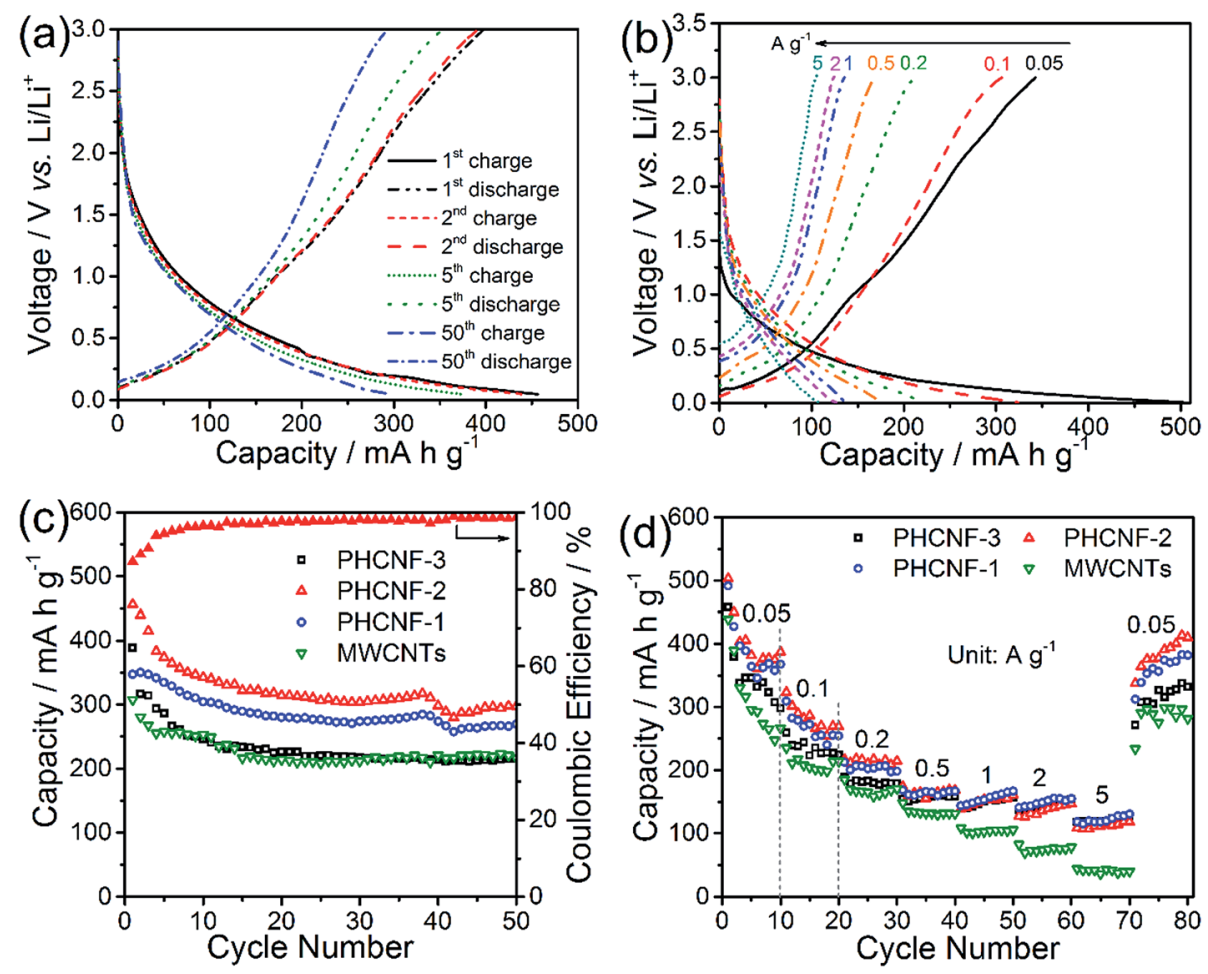

Fig. 4 The electrode of PHCNF-2: (a) voltage profiles after 1st, 2nd, 5th, and 50th cycle at a current density of $0.05 \mathrm{~A} \mathrm{~g}^{-1}$; (b) voltage profiles at the current densities of $0.05,0.1,0.2,0.5,1,2$, and $5 \mathrm{~A} \mathrm{~g}^{-1}$. The electrodes of MWCNTs, PHCNF-1, PHCNF-2, and PHCNF-3: (c) Cycling stability at a current density of $0.05 \mathrm{~A} \mathrm{~g}^{-1}$. (d) Rate performance at the current densities of $0.05,0.1,0.2,0.5,1,2,5$, and $0.05 \mathrm{~A} \mathrm{~g}^{-1}$.

at the same rate. At an extremely high current density of $5 \mathrm{Ag}^{-1}$, the PHCNF-2 electrode is still able to provide a relatively high reversible discharge capacity of $118.1 \mathrm{~mA} \mathrm{~h} \mathrm{~g}{ }^{-1}$, while the pristine MWCNT electrode only gives a reversible capacity as low as $40.3 \mathrm{~mA} \mathrm{~h}^{-1}$. From these results, it should be noted that all the PHCNFs outperform the pristine MWCNTs in rate performances at high current densities of 1,2 , or $5 \mathrm{~A} \mathrm{~g}^{-1}$. This phenomenon may be caused by the favorable porous structures, relatively large specific surface areas of PHCNFs, which facilitates electrolyte diffusion, as well as lithium-ion intercalation and diffusion. When the rate turns back to $0.05 \mathrm{~A} \mathrm{~g}^{-1}$, MWCNTs, PHCNF-1, PHCNF-2 and PHCNF-3 deliver the final reversible capacities of 281.5, 382.1, 410.2, and $331.9 \mathrm{~mA} \mathrm{~h} \mathrm{~g}^{-1}$, respectively. Akin to the cycling measurement, rate performances of the PHCNF-2 electrodes are absolutely much better than that of the MWCNT electrode, which can be ascribed to merits of the non-graphitic carbon shells encapsulated on MWCNTs with a favorable mesopore structure. ${ }^{35-38}$
To further understand the effect of shell thickness on the lithium storage capability, all the samples are analyzed by EIS at the open circuit voltage. Fig. 5 shows that all the plots are composed of two semicircles and s straight sloping line. The possible equivalent circuit model is depicted in the inset for analyzing the Nyquist impedance plots. In the model, the resistances associated with the lithium ion diffusion, the SEI film and the electrode-electrolyte interface are represented by $R_{\mathrm{e}}$ (the straight slopping line at low frequency), $R_{\mathrm{f}}$ (the first semicircle in the high frequency region), and $R_{\text {ct }}$ (the second semicircle in the medium frequency region), respectively. According to the summarized values in Table 3, the electrode of PHCNF-2 has the largest contact impedance $\mathrm{R}_{\mathrm{f}}(82.8 \Omega)$, and the smallest charge transfer impedance $R_{\text {ct }}(111.0 \Omega)$ at OCP, which could be due to its large specific surface area, and advantageous mesopore structure serving as efficient pathway for transport of electrons and lithium ions, respectively. PHCNF-3 exhibits the smallest contact impedance $R_{\mathrm{f}}(1.1 \Omega)$ due to its adverse 


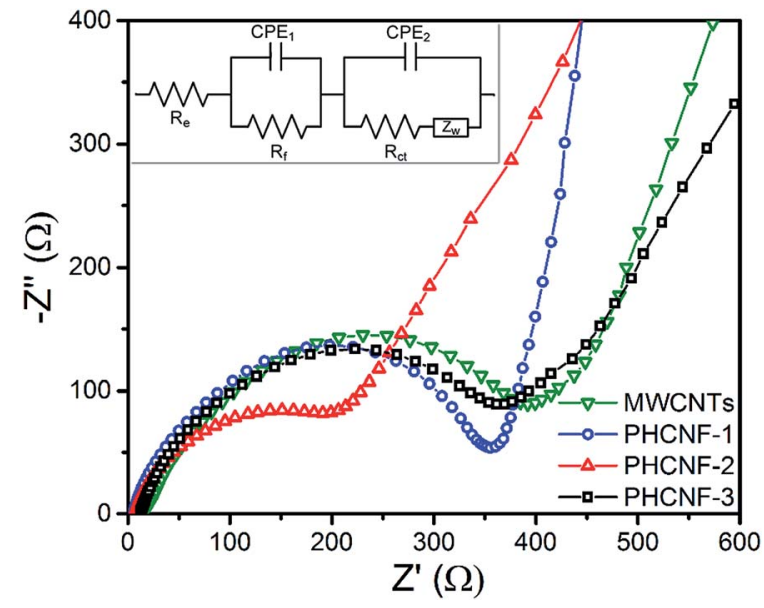

Fig. 5 Nyquist impedance plots for the electrodes of MWCNTs, PHCNF-1, PHCNF-2, and PHCNF-3 at the open circuit voltage. The inset is the equivalent circuit of the $A C$ impedance.

Table 3 Impedance parameters of MWCNTs and PHCNFs

\begin{tabular}{lrrr}
\hline Sample & $R_{\mathrm{e}}(\Omega)$ & $R_{\mathrm{f}}(\Omega)$ & $R_{\mathrm{ct}}(\Omega)$ \\
\hline MWCNTs & 10.9 & 7.9 & 430.0 \\
PHCNF-1 & 5.7 & 51.5 & 298.9 \\
PHCNF-2 & 7.0 & 82.8 & 111.0 \\
PHCNF-3 & 10.8 & 1.1 & 383.6
\end{tabular}

micropore structure resulting in the smallest real contact area and the resultant difficulties of electrolyte diffusion, as well as lithium-ion intercalation and diffusion although it has the largest BET specific surface area. According to EIS method, values of lithium ion diffusion coefficient $D_{\mathrm{Li}}$ for MWCNTs, PHCNF-1, PHCNF-2, and PHCNF-3 are estimated to be $4.36 \times$ $10^{-11}, 2.51 \times 10^{-11}, 1.79 \times 10^{-10}$, and $3.82 \times 10^{-11} \mathrm{~cm}^{2} \mathrm{~s}^{-1}$, respectively (see details in the ESI $\dagger$ ). That is, $D_{\mathrm{Li}}$ value of PHCNF-2 is much higher than MWCNTs and other PHCNFs. Overall, the low charge transfer resistance and the high lithium ion diffusion coefficient of PHCNF-2, mostly resulted from its favorable mesoporous structure and relatively large specific surface area, indicate the good electronic conductivity and high lithium ion transfer speed across interfaces between the electrolyte and active electrode materials, which therefore enhances discharge-charge performance and rate capability of LIBs.

With respect to the pristine MWCNTs with a tube wall thickness of $\sim 10 \mathrm{~nm}$, the as-prepared PHCNFs incorporate the disordered and non-graphitic carbon shells which double or even triple the BET specific surface areas, increase the amount of mesopores by $50 \%$, as well as involve many favorable surface functional groups such as $\mathrm{C}=\mathrm{C}$, hydroxyl and carboxylic groups (Fig. S1 and $\mathrm{S} 2 \dagger$ ). When the shell thickness reaches $\sim 5 \mathrm{~nm}$, PHCNF-2 delivers an enhanced discharge specific capacity of $297.3 \mathrm{~mA} \mathrm{~h} \mathrm{~g}^{-1}$ at a current density of $0.05 \mathrm{~A} \mathrm{~g}^{-1}$ after 50 cycles, a $38 \%$ increase compared to $215.8 \mathrm{~mA} \mathrm{~h} \mathrm{~g}^{-1}$ of the pristine MWCNTs. More favorably, PHCNF-2 exhibits a much-improved discharge specific capacity of $118.1 \mathrm{~mA} \mathrm{~h} \mathrm{~g}^{-1}$ at a high current density of $5 \mathrm{~A} \mathrm{~g}^{-1}$, nearly threefold to $40.3 \mathrm{~mA} \mathrm{~h} \mathrm{~g}{ }^{-1}$ of the pristine MWCNTs. But, if the shell thickness reaches to $\sim 13.5 \mathrm{~nm}$, PHCNF-3 will incorporate excessive micropores on the tube surface which will badly block the lithium-ion intercalation and diffusion, and eventually deteriorate the electrochemical properties.

Compared with the published methods like acid oxidation, ball milling, cutting, drilling holes and opening cap, our method does not damage the microstructure of MWCNTs, which makes it more favorable in environmental protection ( $v s$. acid oxidation and drilling holes) and tailoring CNT arrays for flexible LIBs ( $v s$. ball milling, cutting, and opening cap). Indeed, our method to overcoat a non-graphitic carbon shell is an expansion to tailor the pristine MWCNTs for improving their electrochemical properties, which may further push material sciences development in the field of CNTs.

\section{Conclusions}

Porous hollow carbon nanofibers with tunable shell thicknesses from 2.5 to $13.5 \mathrm{~nm}$ have been synthesized via a hydrothermal approach by using MWCNTs as the scaffolds and sucrose as carbon source. The porous and non-graphitic carbon shells are validated by XRD, Raman spectra, HRTEM, and BET adsorption isotherms. It has been proved that the variations of shell microstructures such as the thickness and the pore structure have considerable impacts on the lithium storage performances when evaluated as the anode in coin-type LIBs. The electrode of PHCNF-2 (shell thickness: $\sim 5.0 \mathrm{~nm}$ ) delivers a specific capacity of $293.7 \mathrm{~mA} \mathrm{~h} \mathrm{~g}^{-1}$ at a current density of $0.05 \mathrm{~A} \mathrm{~g}^{-1}$ after 50 cycles, which is higher than $215.8 \mathrm{~mA} \mathrm{~h} \mathrm{~g}^{-1}$ of the pristine MWCNTs. In brief, the current results demonstrate that overcoating of a thin, porous, and non-graphitic carbon layer on the pristine MWCNTs held a great potential for enhancing their anode performance for LIBs.

\section{Acknowledgements}

This work was financially supported by the NNSF of China (51402242).

\section{References}

1 B. J. Landi, M. J. Ganter, C. D. Cress, R. A. DiLeo and R. P. Raffaelle, Energy Environ. Sci., 2009, 2, 638-654.

2 C. de las Casas and W. Li, J. Power Sources, 2012, 208, 74-85. 3 Z. Yang, J. Ren, Z. Zhang, X. Chen, G. Guan, L. Qiu, Y. Zhang. and H. Peng, Chem. Rev., 2015, 115, 5159-5223.

4 Y. Chen, X. Li, X. Zhou, H. Yao, H. Huang, Y.-W. Mai and L. Zhou, Energy Environ. Sci., 2014, 7, 2689-2696.

5 W. Li, M. Li, M. Wang, L. Zeng and Y. Yu, Nano Energy, 2015, 13, 693-701.

6 J. Jin, Z.-q. Shi and C.-y. Wang, Electrochim. Acta, 2014, 141, 302-310.

7 J. Zhu, C. Chen, Y. Lu, Y. Ge, H. Jiang, K. Fu and X. Zhang, Carbon, 2015, 94, 189-195. 
8 W. Wang, Y. Sun, B. Liu, S. Wang and M. Cao, Carbon, 2015, 91, 56-65.

9 C. Kang, M. Patel, B. Rangasamy, K.-N. Jung, C. Xia, S. Shi and W. Choi, J. Power Sources, 2015, 299, 465-471.

10 B. Zhang, J. Huang and J.-K. Kim, Adv. Funct. Mater., 2015, 25, 5222-5228.

11 F. Yao, D. T. Pham and Y. H. Lee, ChemSusChem, 2015, 8, 2284-2311.

12 M. Ye, C. Hu, L. Lv and L. Qu, J. Power Sources, 2016, 305, 106-114.

13 J. Y. Eom, H. S. Kwon, J. Liu and O. Zhou, Carbon, 2004, 42, 2589-2596.

14 S. W. Lee, B. M. Gallant, Y. Lee, N. Yoshida, D. Y. Kim, Y. Yamada, S. Noda, A. Yamada and Y. Shao-Horn, Energy Environ. Sci., 2012, 5, 5437-5444.

15 Z. Yang, Q. Wang, J. Wang, Z. Li and S. Sang, Mater. Lett., 2007, 61, 3103-3105.

16 J. Eom, D. Kim and H. Kwon, J. Power Sources, 2006, 157, 507-514.

17 X. Gu, C.-j. Tong, B. Wen, L.-m. Liu, C. Lai and S. Zhang, Electrochim. Acta, 2016, 196, 369-376.

18 T. Tao, M. M. Rahman, T. Ramireddy, J. Sunarso, Y. Chen and A. M. Glushenkov, RSC Adv., 2014, 4, 36649-36655.

19 X. X. Wang, J. N. Wang, H. Chang and Y. F. Zhang, Adv. Funct. Mater., 2007, 17, 3613-3618.

20 Q. Zhang, X. Wang, W. Lu, F. Tang, J. Guo, W. Yu, M. Qu and Z. Yu, Mater. Res. Bull., 2013, 48, 2865-2870.

21 H. S. Oktaviano, K. Yamada and K. Waki, J. Mater. Chem., 2012, 22, 25167-25173.

22 Z.-h. Yang and H.-q. Wu, Solid State Ionics, 2001, 143, 173180.

23 M. Wu, J. Chen, C. Wang, F. Wang and B. Yi, Electrochim. Acta, 2013, 105, 462-467.
24 R. Yang, X. Qiu, H. Zhang, J. Li, W. Zhu, Z. Wang, X. Huang and L. Chen, Carbon, 2005, 43, 11-16.

25 Y.-T. Peng and C.-T. Lo, J. Electrochem. Soc., 2015, 162, A1085-A1093.

26 B.-S. Lee, S.-B. Son, K.-M. Park, W.-R. Yu, K.-H. Oh and S.-H. Lee, J. Power Sources, 2012, 199, 53-60.

27 B.-S. Lee, S.-B. Son, K.-M. Park, G. Lee, K. H. Oh, S.-H. Lee and W.-R. Yu, ACS Appl. Mater. Interfaces, 2012, 4, 6702-6710.

28 M. Lallave, J. Bedia, R. Ruiz-Rosas, J. Rodríguez-Mirasol, T. Cordero, J. C. Otero, M. Marquez, A. Barrero and I. G. Loscertales, Adv. Mater., 2007, 19, 4292-4296.

29 L.-X. Yuan, Z.-H. Wang, W.-X. Zhang, X.-L. Hu, J.-T. Chen, Y.-H. Huang and J. B. Goodenough, Energy Environ. Sci., 2011, 4, 269-284.

30 L. Roldán, I. Santos, S. Armenise, J. M. Fraile and E. GarcíaBordejé, Carbon, 2012, 50, 1363-1372.

31 H. Zhao, H.-J. Cui and M.-L. Fu, J. Colloid Interface Sci., 2016, 461, 20-24.

32 X. Zhang, S. Han, P. Xiao, C. Fan and W. Zhang, Carbon, 2016, 100, 600-607.

33 J. Yang, X.-y. Zhou, J. Li, Y.-l. Zou and J.-j. Tang, Mater. Chem. Phys., 2012, 135, 445-450.

34 Y. Yang, K. Wu, R. Pang, X. Zhou, Y. Zhang, X. Wu, C. Wu, H. Wu and S. Guo, RSC Adv., 2013, 3, 14016-14020.

35 J. Li, L. Wang, X. He and J. Wang, ACS Sustainable Chem. Eng., 2016, 4, 4217-4223.

36 H. Lee, J.-K. Yoo, J.-H. Park, J. H. Kim, K. Kang and Y. S. Jung, Adv. Energy Mater., 2012, 2, 914.

37 J.-G. Lee, D. Son, C. Kim and B. Park, J. Power Sources, 2007, 172, 908-912.

38 X. Yang, H. Huang, G. Zhang, X. Li, D. Wu and R. Fu, Mater. Chem. Phys., 2015, 149-150, 657-662. 